\title{
Chromoendoscopic findings useful for diagnosis and assessment of treatments for ileac lesions of Henoch-Schönlein purpura
}
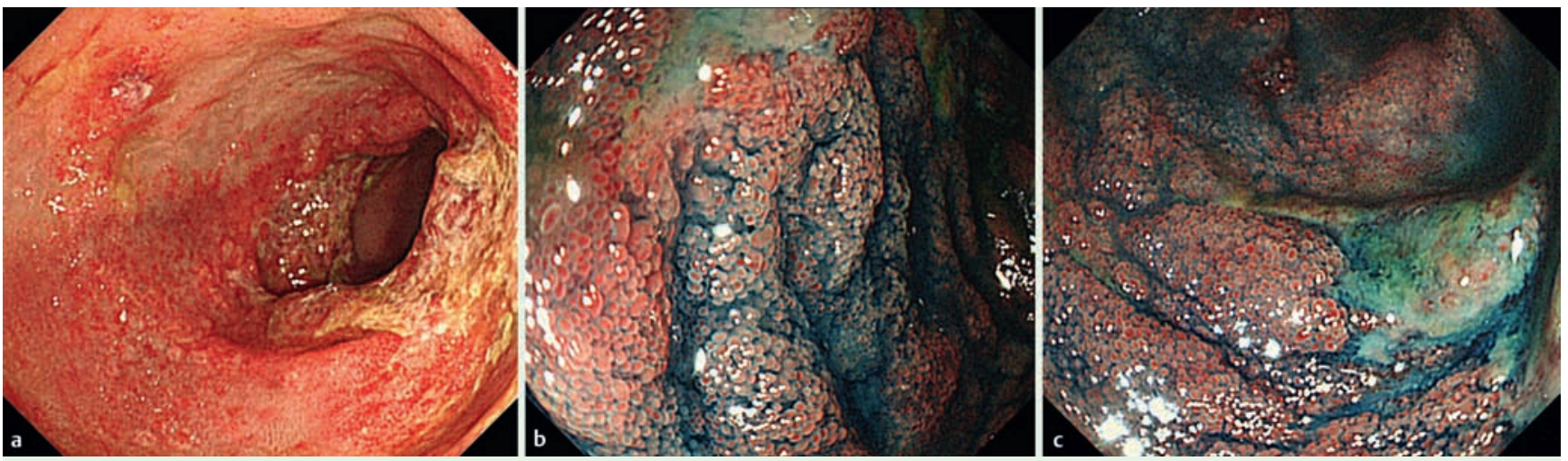

Fig. 1 Case 1. a Total colonoscopy showing severe diffuse redness and insular, fur-coated, shallow ulcers in the terminal ileum. b Chromoendoscopic view with indigo carmine contrast demonstrates redness of the intestinal villi (similar to the so-called "hemorrhagic blebs"). $\mathbf{c}$ The boundary between the inflamed villi and the ulcerations is clearly visualized on the contrast view.
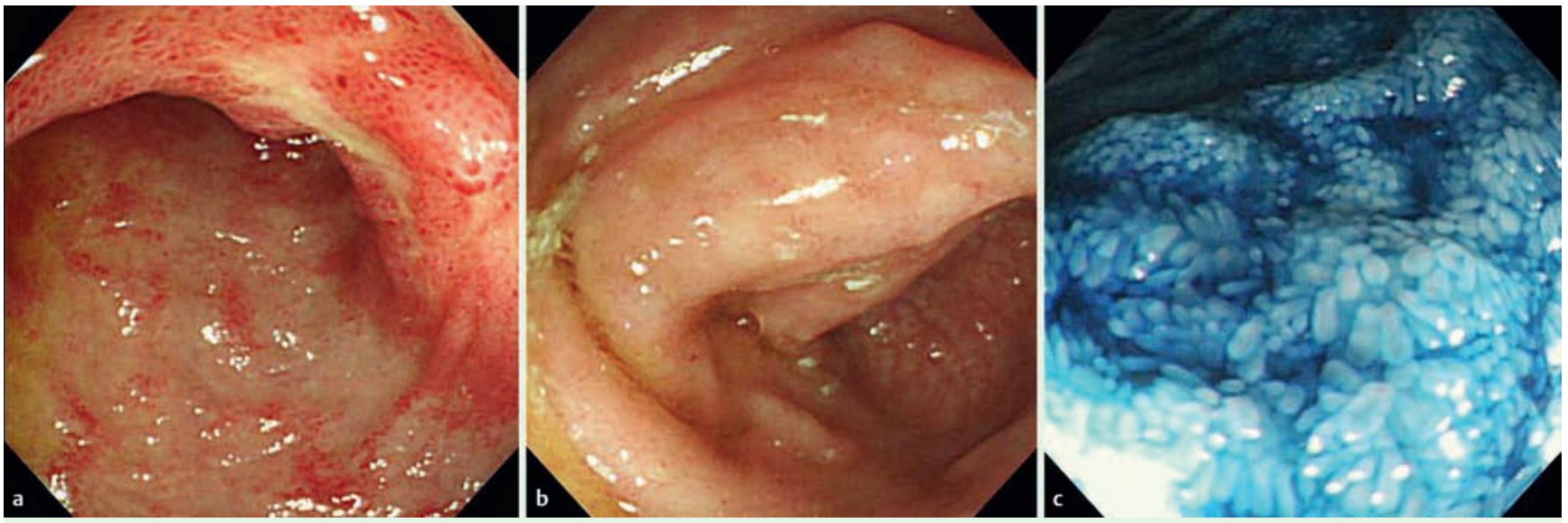

Fig. 2 Case 2. a Total colonoscopy view: there is extensive redness of the terminal ileum, consisting of granular "hemorrhagic blebs", discrete ulcers, and severe mucosal edema. The villous structure of the ileac mucosa appears to be atrophied or sloughed off. $\mathbf{b}$ Conventional colonoscopy following treatment with prednisolone. Note the absence of redness, ulcers, and edema. c Chromoendoscopy following treatment with prednisolone. This view clearly shows the disappearance of redness as well as the regeneration of the mucosal villi.

Henoch-Schönlein purpura (HSP) is a systemic disease characterized by the deposition of immune complexes and involving multiple organs [1]. One of the organs commonly affected is the gastrointestinal tract. Since approximately 15\% of patients with HSP develop purpura after the appearance of digestive symptoms [2], early diagnosis by detection of gastrointestinal lesions using endoscopy and immediate treatment are necessary. Here, we report two cases of HSP examined by chromoendoscopy with indigo carmine contrast. This procedure clearly delineates the endoscopic features of active ileac lesions of HSP, and has also been proven as useful for treatment assessment.

\section{Case 1 \\ $\nabla$}

A 32-year-old man attended the hospital for lower abdominal pain and diarrhea, accompanied by purpura of the shanks. Urgent total colonoscopy revealed severe diffuse redness in the terminal ileum, along with shallow, insular ulcers with a fur coating ( $\bullet$ Fig. 1 a). The chromoendoscopic view with indigo carmine contrast demonstrated redness in each intestinal villus, thought to be equivalent to the so- called "hemorrhagic bleb" ( $\bullet$ Fig. 1 b), a typical endoscopic feature of an active intestinal lesion in HSP [3]. On the other hand, the contrast view clearly delineated the margin between the inflamed villi and the ulcerations ( $\bullet$ Fig. $1 \mathrm{c}$ ).

\section{Case 2}

$\nabla$

A 27-year-old man attended the hospital for lower abdominal pain. Total colonoscopy showed widespread redness, consisting of granular "hemorrhagic blebs", discrete ulcers, and severe mucosal edema in the terminal ileum ( $\bullet$ Fig. 2 a). Be- 
cause of the severe inflammation, the villi in the ileac mucosal layer seemed to be atrophied or sloughed off. Colonoscopy was performed 14 days after prednisolone administration, and the redness, ulcers, and edema were found to have nearly disappeared (as observed in the conventional view, Fig. 2 b). The chromoendoscopic view with indigo carmine contrast clearly demonstrated not only the disappearance of redness but also the regeneration of the villous structure (० Fig. 2c).

Endoscopy_UCTN_Code_CCL_1AD_2AF
S. Oomori ${ }^{1}$, K. Abe ${ }^{2}$, S. Takagi ${ }^{3}$

1 Department of Gastroenterology, Japanese Red Cross Sendai Hospital, Sendai, Japan

2 Department of Pathology, Japanese Red Cross Sendai Hospital, Sendai, Japan

3 Department of Gastroenterology, Iwate Prefectural Iwai Hospital, Ichinoseki, Japan

\section{References}

1 Szer IS. Henoch-Schönlein purpura. Curr Opin Rheumatol 1994; 6: 25-31

2 Auringer ST, Scharling ES, Sumner TE. CT of the pediatric gastrointestinal tract. Radiol Clin North Am 1996; 34: 701 - 715

3 Nishiyama R, Nakajima $N$, Ogihara A et al. Endoscope images of Schonlein-Henoch purpura. Digestion 2008; 77: 236-241
Bibliography

DOI $10.1055 / \mathrm{s}-0029-1214627$

Endoscopy 2009; 41: E111-E112

(c) Georg Thieme Verlag KG Stuttgart · New York . ISSN 0013-726X

Corresponding author

\section{S. Oomori}

Department of Gastroenterology, Japanese Red Cross Sendai Hospital 2-43-3 Yagiyama-honcho

Taihaku-ku

Sendai

Miyagi Prefecture 982-8501

Japan

Fax: +81-22-2431101

goodluck-ohmori@mta.biglobe.ne.jp 